\title{
Periodicity of hyperplane arrangements with integral coefficients modulo positive integers
}

\author{
Hidehiko Kamiya • Akimichi Takemura • \\ Hiroaki Terao
}

Received: 5 April 2007 / Accepted: 24 July 2007 / Published online: 25 August 2007

(C) Springer Science+Business Media, LLC 2007

\begin{abstract}
We study central hyperplane arrangements with integral coefficients modulo positive integers $q$. We prove that the cardinality of the complement of the hyperplanes is a quasi-polynomial in two ways, first via the theory of elementary divisors and then via the theory of the Ehrhart quasi-polynomials. This result is useful for determining the characteristic polynomial of the corresponding real arrangement. With the former approach, we also prove that intersection lattices modulo $q$ are periodic except for a finite number of $q$ 's.
\end{abstract}

Keywords Characteristic polynomial $\cdot$ Ehrhart quasi-polynomial $\cdot$ Elementary divisor · Hyperplane arrangement · Intersection lattice

\section{Introduction}

When a linear form in $x_{1}, \ldots, x_{m}$ with integral coefficients is given, we may naturally consider its " $q$-reduction" for any positive integer $q$. The $q$-reduction is the image by the modulo $q$ projection $\mathbb{Z}\left[x_{1}, \ldots, x_{m}\right] \longrightarrow \mathbb{Z}_{q}\left[x_{1}, \ldots, x_{m}\right]$, where $\mathbb{Z}_{q}=\mathbb{Z} / q \mathbb{Z}$. In this paper, we call the kernel of the resulting linear form a "hyperplane" in $V:=\mathbb{Z}_{q}^{m}$. Suppose that a finite set of nonzero linear forms with integral coefficients is given. Then it not only defines a central hyperplane arrangement $\mathcal{A}$ in $\mathbb{R}^{m}$, but also gives

This work was supported by the MEXT and the JSPS.

H. Kamiya

Faculty of Economics, Okayama University, Okayama, Japan

\section{A. Takemura}

Graduate School of Information Science and Technology, University of Tokyo, Tokyo, Japan

H. Terao (ه)

Department of Mathematics, Hokkaido University, Hokkaido, Japan

e-mail: hterao00@za3.so-net.ne.jp 
a "hyperplane arrangement" $\mathcal{A}_{q}$ in $V$ through the $q$-reduction for each $q \in \mathbb{Z}_{>0}$. A basic fact we prove in this paper is that the cardinality of the complement $M\left(\mathcal{A}_{q}\right)$ of the arrangement $\mathcal{A}_{q}$ in $V$, as a function of $q$, is a quasi-polynomial in $q$. (In other words, there exist a positive integer $\rho$ (a period) and polynomials $P_{r}(t)(1 \leq r \leq \rho)$ such that $\left|M\left(\mathcal{A}_{q}\right)\right|=P_{r}(q)\left(q \in r+\rho \mathbb{Z}_{\geq 0}\right)$ for all $q \in \mathbb{Z}_{>0}$. $)$ We provide two proofs of this fact. The first proof uses the theory of elementary divisors. The second proof is based on the theory of the Ehrhart quasi-polynomials applied to each chamber of the arrangement.

In our setting, the approach via elementary divisors is more powerful than the one via the Ehrhart theory. The former gives more information on the coefficients of the quasi-polynomials, and it also enables us to prove that the intersection lattices modulo $q$ are themselves periodic except for a finite number of $q$ 's. Despite the advantage of the approach via elementary divisors for our setting, we also consider the connection to the Ehrhart theory an important aspect of our discussion, because many results in the Ehrhart theory can be applied to further develop the arguments in this paper.

Especially when $q$ is a prime, the arrangement $\mathcal{A}_{q}$ lies in the vector space $V=\mathbb{Z}_{q}^{m}$. In this case, it is well known (e.g., [9], [16, (4.10)], [10, Theorem 3.2]) that $\left|M\left(\mathcal{A}_{q}\right)\right|$ is equal to $\chi\left(\mathcal{A}_{q}, q\right)$ and that $\chi\left(\mathcal{A}_{q}, t\right)$ coincides with $\chi(\mathcal{A}, t)$ for a sufficiently large prime $q$, where $\chi(-, t)$ stands for the characteristic polynomial (e.g., [13, Definition 2.52], [15, Chap. 3, Exercise 56]) of an arrangement. These facts provide the "finite field method" to study the real arrangement $\mathcal{A}$. The method was initiated and systematically applied by Athanasiadis [1-3]. It has been used to solve problems related to hyperplane arrangements by Björner and Ekedahl [7], and Blass and Sagan [8] among others. It was also used in [10] to find the characteristic polynomials of the mid-hyperplane arrangements up to a certain dimension. Athanasiadis [4] studies a problem similar to but different from the problem in the present paper. He proves that the coefficients of the characteristic polynomial of a certain deformation of a central arrangement are quasi-polynomials. A series of works by Athanasiadis on the finite field method is worth a special mention as the driving force of the research on this method.

For the theory of hyperplane arrangements, the reader is referred to [13]. For the Ehrhart theory for counting lattice points in rational polytopes, see the book by Beck and Robins [5]. Beck and Zaslavsky [6] study the extension of the Ehrhart theory to counting lattice points in "inside-out polytopes."

The organization of the paper is as follows. In the rest of this section, we set up our notation. In Section 2, we prove that the cardinality of the complement $M\left(\mathcal{A}_{q}\right)$ is a quasi-polynomial in $q$, via the theory of elementary divisors (Section 2.1) and via the theory of the Ehrhart quasi-polynomials (Section 2.2). Based on this result, we consider a way of calculating the characteristic polynomial $\chi(\mathcal{A}, t)$ of the corresponding real arrangement $\mathcal{A}$ (Section 2.3). In Section 3, we prove that the intersection lattices modulo $q$ are periodic except for a finite number of $q$ 's.

In our forthcoming paper [11], we discuss the results in the present paper for the arrangements arising from root systems and the mid-hyperplane arrangements. 


\subsection{Setup and notation}

Let $m, n \in \mathbb{Z}_{>0}$ be positive integers. In this paper, $m$ denotes the dimension and $n$ is the number of hyperplanes in an arrangement. Suppose we are given an $m \times n$ integer matrix

$$
C=\left(c_{1}, \ldots, c_{n}\right) \in \operatorname{Mat}_{m \times n}(\mathbb{Z})
$$

consisting of column vectors $c_{j}=\left(c_{1 j}, \ldots, c_{m j}\right)^{T} \in \mathbb{Z}^{m}, 1 \leq j \leq n$. Here, ${ }^{T}$ denotes the transpose and $\operatorname{Mat}_{m \times n}(\mathbb{Z})$ stands for the set of $m \times n$ matrices with integer elements. We assume that integral vectors $c_{j}$ are nonzero:

$$
c_{j} \neq(0, \ldots, 0)^{T}, \quad 1 \leq j \leq n .
$$

Consider a real central hyperplane arrangement

$$
\mathcal{A}=\mathcal{A}_{C}:=\left\{H_{j}: 1 \leq j \leq n\right\}
$$

with

$$
H_{j}=H_{c_{j}}:=\left\{x=\left(x_{1}, \ldots, x_{m}\right) \in \mathbb{R}^{m}: x c_{j}=0\right\} .
$$

As an example, let us take $m=2, n=3$ and

$$
C=\left(\begin{array}{ccc}
1 & 1 & -2 \\
-1 & 1 & 1
\end{array}\right),
$$

i.e., $c_{1}=(1,-1)^{T}, c_{2}=(1,1)^{T}, c_{3}=(-2,1)^{T}$. Then the corresponding hyperplane arrangement in $\mathbb{R}^{2}=\{(x, y): x, y \in \mathbb{R}\}$ is $\mathcal{A}=\left\{H_{1}, H_{2}, H_{3}\right\}$ with

$$
H_{1}: x-y=0, \quad H_{2}: x+y=0, \quad H_{3}:-2 x+y=0 .
$$

Since the coefficient vectors $c_{j}=\left(c_{1 j}, \ldots, c_{m j}\right)^{T} \in \mathbb{Z}^{m}, 1 \leq j \leq n$, defining $H_{j}$ are integral, we can consider the reductions of $c_{j}$ modulo positive integers $q \in \mathbb{Z}_{>0}$. Fix $q \in \mathbb{Z}_{>0}$ and let

$$
\left[c_{j}\right]_{q}=\left(\left[c_{1 j}\right]_{q}, \ldots,\left[c_{m j}\right]_{q}\right)^{T} \in \mathbb{Z}_{q}^{m}
$$

be the $q$-reduction of $c_{j}$, i.e., $\left[c_{i j}\right]_{q}=c_{i j}+q \mathbb{Z} \in \mathbb{Z}_{q}, 1 \leq i \leq m, 1 \leq j \leq n$. In $V=\mathbb{Z}_{q}^{m}$, let us consider

$$
H_{j, q}=H_{c_{j}, q}:=\left\{x=\left(x_{1}, \ldots, x_{m}\right) \in V: x\left[c_{j}\right]_{q}=[0]_{q}\right\}
$$

and define

$$
\mathcal{A}_{q}=\mathcal{A}_{C, q}:=\left\{H_{j, q}: 1 \leq j \leq n\right\} .
$$

We emphasize that $\mathcal{A}_{q}=\mathcal{A}_{C, q}$ is determined by $C$ and $q$, but not by $\mathcal{A}=\mathcal{A}_{C}$ and $q$. For a non-prime $q$, it may not be appropriate to call $H_{j, q}$ a hyperplane, but by abusing 
the terminology we call $H_{j, q}$ a hyperplane, and $\mathcal{A}_{q}$ an arrangement of hyperplanes. In our previous example (2), $\mathcal{A}_{q}=\left\{H_{1, q}, H_{2, q}, H_{3, q}\right\}$ with

$$
\begin{aligned}
H_{1, q} & =\left\{\left([0]_{q},[0]_{q}\right),\left([1]_{q},[1]_{q}\right), \ldots,\left([q-1]_{q},[q-1]_{q}\right)\right\} \\
H_{2, q} & =\left\{\left([0]_{q},[0]_{q}\right),\left([1]_{q},[q-1]_{q}\right), \ldots,\left([q-1]_{q},[1]_{q}\right)\right\}, \\
H_{3, q} & =\left\{\left([0]_{q},[0]_{q}\right),\left([1]_{q},[2]_{q}\right),\left([2]_{q},[4]_{q}\right), \ldots,\left([q-1]_{q},[q-2]_{q}\right)\right\} .
\end{aligned}
$$

In the finite field method and its generalization in the present paper, we are interested in the cardinality of the complement of $\mathcal{A}_{q}$. We denote the complement by

$$
M\left(\mathcal{A}_{q}\right):=V \backslash \bigcup_{1 \leq j \leq n} H_{j, q}
$$

and its cardinality by $\left|M\left(\mathcal{A}_{q}\right)\right|$. We will prove that $\left|M\left(\mathcal{A}_{q}\right)\right|$ is an integral quasipolynomial in $q$ of degree $m$ and with the leading coefficient identically equal to 1 . That is, there exist a period $\rho \in \mathbb{Z}_{>0}$ and monic integral polynomials $P_{1}(t), \ldots, P_{\rho}(t) \in \mathbb{Z}[t]$ of degree $m$ such that

$$
\left|M\left(\mathcal{A}_{q}\right)\right|=P_{r}(q) \quad\left(q \in \mathbb{Z}_{>0}, 1 \leq r \leq \rho,[q]_{\rho}=[r]_{\rho}\right) .
$$

In this paper, we will call (4) the characteristic quasi-polynomial of $\mathcal{A}_{q}$, because, as we will see in Section 2.3, the value (4) coincides with $\chi(\mathcal{A}, q)$ if $q$ and $\rho$ are coprime, where $\chi(\mathcal{A}, t)$ denotes the characteristic polynomial (e.g., [13, Definition 2.52], [15, Chap. 3, Exercise 56]) of the real arrangement $\mathcal{A}$. The minimum period is simply called the period of $\left|M\left(\mathcal{A}_{q}\right)\right|$. Often it is not trivial to find the period of $\left|M\left(\mathcal{A}_{q}\right)\right|$, although it is relatively easy to evaluate some multiple of the period, which we simply call a period.

This is because of the following. The sum $\chi_{1}(q)+\chi_{2}(q)$ of two quasi-polynomials $\chi_{1}(q), \chi_{2}(q)$ is a quasi-polynomial having as a period the least common multiple of the periods of $\chi_{1}(q)$ and $\chi_{2}(q)$. However, due to possible cancellations of terms, the period of $\chi_{1}(q)+\chi_{2}(q)$ may be smaller than this least common multiple. See McAllister and Woods [12].

For a subset $J=\left\{j_{1}, \ldots, j_{k}\right\} \subseteq\{1, \ldots, n\}$, write

$$
H_{J, q}:=\bigcap_{j \in J} H_{j, q}=H_{j_{1}, q} \cap \cdots \cap H_{j_{k}, q} .
$$

When $J$ is nonempty, $H_{J, q}$ in (5) is determined by the $q$-reduction of the $m \times k$ submatrix

$$
C_{J}:=\left(c_{j_{1}}, \ldots, c_{j_{k}}\right) \in \mathrm{Mat}_{m \times k}(\mathbb{Z})
$$

of $C$; when $J$ is empty, we understand that $H_{\emptyset, q}=V$.

The Smith normal form of an integer matrix $G \in \operatorname{Mat}_{m \times k}(\mathbb{Z}), k \in \mathbb{Z}_{>0}$, is

$$
\begin{aligned}
& S G T=\left(\begin{array}{cc}
E & O \\
O & O
\end{array}\right) \in \operatorname{Mat}_{m \times k}(\mathbb{Z}), \quad E=\operatorname{diag}\left(e_{1}, \ldots, e_{\ell}\right), \quad \ell=\operatorname{rank} G, \\
& e_{1}, \ldots, e_{\ell} \in \mathbb{Z}_{>0}, \quad e_{1}\left|e_{2}\right| \cdots \mid e_{\ell},
\end{aligned}
$$


where $S \in \operatorname{Mat}_{m \times m}(\mathbb{Z})$ and $T \in \operatorname{Mat}_{k \times k}(\mathbb{Z})$ are unimodular matrices. The positive integers $e_{1}, \ldots, e_{\ell}$ are the elementary divisors of $G$. For simplicity, we often use the following notation

$$
\operatorname{diag}\left(\left\{e_{1}, \ldots, e_{\ell}\right\} ; m, k\right)=\left(\begin{array}{cc}
E & O \\
O & O
\end{array}\right) \in \operatorname{Mat}_{m \times k}(\mathbb{Z}) .
$$

\section{Characteristic quasi-polynomial}

\subsection{Via elementary divisors}

In this subsection, we prove that $\left|M\left(\mathcal{A}_{q}\right)\right|=\left|V \backslash \bigcup_{1 \leq j \leq n} H_{j, q}\right|$ is a quasi-polynomial in $q \in \mathbb{Z}_{>0}$ using the theory of elementary divisors.

Let $Y(\cdot), Y \subseteq V$, stand for the characteristic function (indicator function) of $Y$ : $Y(x)=1, x \in Y$ and $Y(x)=0, x \in V \backslash Y$. Then for every $x \in V$,

$$
\prod_{j=1}^{n}\left(1-H_{j, q}(x)\right)=\sum_{J \subseteq\{1, \ldots, n\}}(-1)^{|J|} H_{J, q}(x)=V(x)+\sum_{\emptyset \neq J \subseteq\{1, \ldots, n\}}(-1)^{|J|} H_{J, q}(x),
$$

which may be viewed as the inclusion-exclusion principle. Therefore, from the relation $x \in M\left(\mathcal{A}_{q}\right) \Leftrightarrow 1=\prod_{j=1}^{n}\left(1-H_{j, q}(x)\right)$, we have

$$
\left|M\left(\mathcal{A}_{q}\right)\right|=\sum_{x \in V} \prod_{j=1}^{n}\left(1-H_{j, q}(x)\right)=q^{m}+\sum_{\emptyset \neq J \subseteq\{1, \ldots, n\}}(-1)^{|J|}\left|H_{J, q}\right| .
$$

Hence it suffices to verify that for each nonempty subset $J=\left\{j_{1}, \ldots, j_{k}\right\}$ of $\{1, \ldots, n\}$, the cardinality $\left|H_{J, q}\right|$ is a quasi-polynomial in $q \in \mathbb{Z}_{>0}$. Actually, we can show that $\left|H_{J, q}\right|$ is a quasi-monomial with an integral coefficient.

Fix $J=\left\{j_{1}, \ldots, j_{k}\right\} \neq \emptyset$ and consider $C_{J}=\left(c_{j_{1}}, \ldots, c_{j_{k}}\right) \in \operatorname{Mat}_{m \times k}(\mathbb{Z})$. For each $q \in \mathbb{Z}_{>0}$, let us define $f_{J, q}: V=\mathbb{Z}_{q}^{m} \rightarrow \mathbb{Z}_{q}^{k}$ by

$$
x \mapsto x\left[C_{J}\right]_{q},
$$

where $\left[C_{J}\right]_{q}=\left(\left[c_{j_{1}}\right]_{q}, \ldots,\left[c_{j_{k}}\right]_{q}\right) \in \operatorname{Mat}_{m \times k}\left(\mathbb{Z}_{q}\right)$ is the $q$-reduction of $C_{J}$. Then $\left|H_{J, q}\right|=\left|\operatorname{ker} f_{J, q}\right|$, so the problem reduces to proving that $\left|\operatorname{ker} f_{J, q}\right|$ is a quasimonomial in $q$. This fact can be shown by using the following general lemma.

Lemma 2.1 Let $m$ and $k$ be positive integers. Let $f: \mathbb{Z}^{m} \rightarrow \mathbb{Z}^{k}$ be a $\mathbb{Z}$-homomorphism. Then the cardinality of the kernel of the induced morphism $f_{q}: \mathbb{Z}_{q}^{m} \rightarrow \mathbb{Z}_{q}^{k}$ is a quasi-monomial of $q \in \mathbb{Z}_{>0}$. Furthermore, suppose $f$ is represented by a matrix $G \in \operatorname{Mat}_{m \times k}(\mathbb{Z})$. Then this quasi-monomial $\left|\operatorname{ker} f_{q}\right|, q \in \mathbb{Z}_{>0}$, can be expressed as

$$
\left|\operatorname{ker} f_{q}\right|=\left(d_{1}(q) \cdots d_{\ell}(q)\right) q^{m-\ell},
$$

where $\ell=\operatorname{rank} G$ and $d_{j}(q):=\operatorname{gcd}\left\{e_{j}, q\right\}, 1 \leq j \leq \ell$. Here, $e_{1}, \ldots, e_{\ell} \in \mathbb{Z}_{>0}$, $e_{1}\left|e_{2}\right| \cdots \mid e_{\ell}$, are the elementary divisors of $G$. In that case, the quasi-monomial $\left|\operatorname{ker} f_{q}\right|, q \in \mathbb{Z}_{>0}$, has the minimum period $e_{\ell}$, where we consider $e_{0}$ to be one. 
Proof If $f$ is the zero $\mathbb{Z}$-homomorphism, then $\left|\operatorname{ker} f_{q}\right|=\left|\mathbb{Z}_{q}^{m}\right|=q^{m}$ and the theorem is trivially true. So we may assume that $f$ is not the zero $\mathbb{Z}$-homomorphism. Since $\left|\operatorname{ker} f_{q}\right|=q^{m} /\left|\operatorname{im} f_{q}\right|$, we will study $\left|\operatorname{im} f_{q}\right|$.

Suppose $f$ is represented by an $m \times k$ integer matrix $G \in \operatorname{Mat}_{m \times k}(\mathbb{Z})$. Then, for $q \in \mathbb{Z}_{>0}$, the induced morphism $f_{q}: \mathbb{Z}_{q}^{m} \rightarrow \mathbb{Z}_{q}^{k}$ is given by $x \mapsto x[G]_{q}$.

Consider the Smith normal form of $G$ in (6). Since unimodularity is preserved under $q$-reductions, we may assume that $G$ is of the form

$$
G=\operatorname{diag}\left(\left\{e_{1}, \ldots, e_{\ell}\right\} ; m, k\right)
$$

from the outset. Then we have

$$
f_{q}(x)=\left(\left[e_{1}\right]_{q} x_{1}, \ldots,\left[e_{\ell}\right]_{q} x_{\ell},[0]_{q}, \ldots,[0]_{q}\right) \in \mathbb{Z}_{q}^{k}
$$

for $x=\left(x_{1}, \ldots, x_{m}\right) \in \mathbb{Z}_{q}^{m}$. Therefore, $\operatorname{im} f_{q}=\left[e_{1}\right]_{q} \mathbb{Z}_{q} \times \cdots \times\left[e_{\ell}\right]_{q} \mathbb{Z}_{q}$ and hence

$$
\left|\operatorname{im} f_{q}\right|=\frac{q}{d_{1}(q)} \times \cdots \times \frac{q}{d_{\ell}(q)}=\frac{q^{\ell}}{d_{1}(q) \cdots d_{\ell}(q)},
$$

where $d_{j}(q)=\operatorname{gcd}\left\{e_{j}, q\right\}, 1 \leq j \leq \ell$. Consequently, we obtain (9).

Now, for any $j=1, \ldots, \ell$, we have $d_{j}\left(q+e_{\ell}\right)=\operatorname{gcd}\left\{e_{j}, q+e_{\ell}\right\}=\operatorname{gcd}\left\{e_{j}, q\right\}=$ $d_{j}(q)$. Therefore, (9) is a quasi-monomial in $q$ of degree $m-\ell<m$ and with a period $e_{\ell}$. In fact, we can show that $e_{\ell}$ is the minimum period as follows.

Let $e^{\prime}$ be the minimum period. Note that $e^{\prime} \mid e_{\ell}$. We have $d_{j}\left(e_{\ell}\right)=e_{j} \geq d_{j}\left(e^{\prime}\right)=$ $d_{j}\left(e^{\prime}+e_{\ell}\right)>0$ for all $j=1, \ldots, \ell$. Since $e^{\prime}$ is a period, $d_{1}\left(e_{\ell}\right) \cdots d_{\ell}\left(e_{\ell}\right)=d_{1}\left(e^{\prime}+\right.$ $\left.e_{\ell}\right) \cdots d_{\ell}\left(e^{\prime}+e_{\ell}\right)$. Therefore $e_{\ell}=d_{\ell}\left(e_{\ell}\right)=d_{\ell}\left(e^{\prime}+e_{\ell}\right)=e^{\prime}$.

Now, $f_{J, q}: V=\mathbb{Z}_{q}^{m} \rightarrow \mathbb{Z}_{q}^{k}$ in (8) is induced from the $\mathbb{Z}$-homomorphism $f_{J}$ : $\mathbb{Z}^{m} \rightarrow \mathbb{Z}^{k}$ represented by $C_{J}$. Thus, Lemma 2.1 implies that

$$
\left|H_{J, q}\right|=\left|\operatorname{ker} f_{J, q}\right|=\left(d_{J, 1}(q) \cdots d_{J, \ell(J)}(q)\right) q^{m-\ell(J)}
$$

is a quasi-monomial with the period $e_{J, \ell(J)}$, where $\ell(J):=\operatorname{rank} C_{J}$ and $d_{J, j}(q):=$ $\operatorname{gcd}\left\{e_{J, j}, q\right\}, 1 \leq j \leq \ell(J)$. Here, $e_{J, 1}, \ldots, e_{J, \ell(J)} \in \mathbb{Z}_{>0}, e_{J, 1}\left|e_{J, 2}\right| \cdots \mid e_{J, \ell(J)}$, denote the elementary divisors of $C_{J}$. Note that $\ell(J)>0$ for all $J,|J| \geq 1$, because of the assumption (1).

Remark 2.2 Assume that $q$ is prime. Then each $d_{J, j}(q)=\operatorname{gcd}\left\{e_{J, j}, q\right\}, \quad 1 \leq j \leq$ $\ell(J)$, is 1 or $q$, and $d_{J, j}(q)=q$ if and only if $\left[e_{J, j}\right]_{q}=0$. It follows from (10) that $X:=H_{J, q}$ for any nonempty $J$ satisfies $|X|=q^{m-\ell^{\prime}}=q^{\operatorname{dim} X}$, where $\ell^{\prime}=\mid\{j$ : $\left.1 \leq j \leq \ell(J),\left[e_{J, j}\right]_{q} \neq 0\right\} \mid$. Note that $|X|=q^{\operatorname{dim} X}$ for $X=H_{J, q}$ is true also when $J$ is empty: $\left|\mathbb{Z}_{q}^{m}\right|=q^{m}$.

From the discussions so far, we reach the following conclusions. First, $\left|M\left(\mathcal{A}_{q}\right)\right|$, $q \in \mathbb{Z}_{>0}$, is a monic quasi-polynomial in $q$ of degree $m$. Second, a period of this quasi-polynomial can be obtained in the following way. For each $m \times k(1 \leq k \leq n)$ submatrices $C_{J}$ of $C=\left(c_{1}, \ldots, c_{n}\right) \in \operatorname{Mat}_{m \times n}(\mathbb{Z})$, find its largest elementary divisor 
$e(J):=e_{J, \ell(J)}$. Let

$$
\rho_{0}:=\operatorname{lcm}\{e(J): J \subseteq\{1, \ldots, n\}, J \neq \emptyset\} .
$$

Then $\rho_{0}$ is a period of $\left|M\left(\mathcal{A}_{q}\right)\right|$.

For computing $\rho_{0}$ when $m<n$, we can restrict the size of $J$ as $|J| \leq m$ :

$$
\rho_{0}=\operatorname{lcm}\{e(J): J \subseteq\{1, \ldots, n\}, 1 \leq|J| \leq \min \{m, n\}\} .
$$

We can prove (11) in the following way. First, we note the next lemma.

Lemma 2.3 Let $f_{1}, f_{2}: \mathbb{Z}^{n} \rightarrow \mathbb{Z}^{m}$ be two $\mathbb{Z}$-homomorphisms with $\operatorname{rank}\left(\operatorname{im} f_{1}\right)=$ $\operatorname{rank}\left(\operatorname{im} f_{2}\right)$ and $\operatorname{im} f_{2} \subseteq \operatorname{im} f_{1}$. Then the largest elementary divisor of $f_{1}$ divides the largest elementary divisor of $f_{2}$.

Proof Define $I_{i}=\operatorname{Ann}\left(\operatorname{coker} f_{i}\right):=\left\{p \in \mathbb{Z}: p\left(\operatorname{coker} f_{i}\right)=0\right\}$, so the ideal $I_{i}$ is generated by the largest elementary divisor of $f_{i}(i=1,2)$. Since there is a natural projection coker $f_{2} \rightarrow \operatorname{coker} f_{1}$, we have $I_{2} \subseteq I_{1}$. This implies the lemma.

Now, suppose $m<n$, and take an arbitrary $J \subseteq\{1, \ldots, n\}$ with $m<|J| \leq n$. Let $\ell=\ell(J)=\operatorname{rank} C_{J}(\leq m)$. Then we can take a subset $\tilde{J} \subset J,|\tilde{J}|=\ell$, such that $\operatorname{rank} C_{\tilde{J}}=\ell$. For this $\tilde{J}$, we have $\operatorname{img} g_{\tilde{J}} \subseteq \operatorname{img} g_{J}$, where $g_{J}, g_{\tilde{J}}: \mathbb{Z}^{n} \rightarrow \mathbb{Z}^{m}$ are the $\mathbb{Z}$-homomorphisms defined by $C_{J}$ and $C_{\tilde{J}}$, respectively: $g_{J}(x)=\sum_{j \in J} x_{j} c_{j}$, $g_{\tilde{J}}(x)=\sum_{j \in \tilde{J}} x_{j} c_{j}, \quad x=\left(x_{1}, \ldots, x_{n}\right)^{T} \in \mathbb{Z}^{n}$. Then Lemma 2.3 implies that $e(J) \mid e(\tilde{J})$. From this observation, we obtain (11). When $n$ is considerably larger than $m$, the restriction $|J| \leq m$ is computationally very useful.

Let us find a period $\rho_{0}$ for our example (2). Take $J=\{1,2\}$. Then we have

$$
C_{J}=\left(\begin{array}{cc}
1 & 1 \\
-1 & 1
\end{array}\right)
$$

with the Smith normal form $\operatorname{diag}(1,2)$. Hence $e(J)=e_{J, 2}=2$. In a similar manner, we can find $e(J)$ for the other $J$ 's with $1 \leq|J| \leq 2$, and obtain $\rho_{0}=$ $\operatorname{lcm}\{1,1,1,2,1,3\}=6$.

Furthermore, for $|J| \geq 1,1 \leq j \leq \ell(J)$,

$$
d_{J, j}(q)=\operatorname{gcd}\left\{e_{J, j}, q\right\}=\operatorname{gcd}\left\{e_{J, j}, \rho_{0}, q\right\}=\operatorname{gcd}\left\{e_{J, j}, \operatorname{gcd}\left\{\rho_{0}, q\right\}\right\} .
$$

This implies that the coefficient $d_{J, 1}(q) \cdots d_{J, \ell(J)}(q)$ of each monomial $\left|H_{J, q}\right|$, $|J| \geq 1$, in (10) depends on $q$ only through $\operatorname{gcd}\left\{\rho_{0}, q\right\}$. Therefore, the constituents of the quasi-polynomial $\left|M\left(\mathcal{A}_{q}\right)\right|$ in (7) coincide for all $q$ with the same $\operatorname{gcd}\left\{\rho_{0}, q\right\}$.

We summarize the results obtained so far as follows:

Theorem 2.4 The function $\left|M\left(\mathcal{A}_{q}\right)\right|$ is a monic quasi-polynomial in $q \in \mathbb{Z}_{>0}$ of degree $m$ with a period $\rho_{0}$ given in (11). Furthermore, in (4) with $\rho=\rho_{0}$, the monic integral polynomials $P_{r}(1 \leq r \leq \rho)$ of degree $m$ depend on $r$ only through $\operatorname{gcd}\left\{\rho_{0}, r\right\}$. 
Let us find the characteristic quasi-polynomial for our example (2). Since $\rho_{0}=6$, we know by Theorem 2.4 that each of the sets $\{1,5\},\{2,4\},\{3,9\},\{6,12\}$ of values of $q$ determines a constituent of the characteristic quasi-polynomial $\left|M\left(\mathcal{A}_{q}\right)\right|$. For $q=1$, we have $V=H_{1,1}=H_{2,1}=H_{3,1}=\left\{\left([0]_{1},[0]_{1}\right)\right\}$ and thus $\left|M\left(\mathcal{A}_{1}\right)\right|=0$. For $q=5$, we can count $\left|H_{1,5} \cup H_{2,5} \cup H_{3,5}\right|=13$ and get $\left|M\left(\mathcal{A}_{5}\right)\right|=5^{2}-13=12$. By interpolation, we obtain the constituent $q^{2}-3 q+2$ for $\operatorname{gcd}\{6, q\}=1$. In this way, we can get the following characteristic quasi-polynomial:

$$
\left|M\left(\mathcal{A}_{q}\right)\right|= \begin{cases}q^{2}-3 q+2 & \text { when } \operatorname{gcd}\{6, q\}=1 \\ q^{2}-3 q+3 & \text { when } \operatorname{gcd}\{6, q\}=2 \\ q^{2}-3 q+4 & \text { when } \operatorname{gcd}\{6, q\}=3 \\ q^{2}-3 q+5 & \text { when } \operatorname{gcd}\{6, q\}=6\end{cases}
$$

From this characteristic quasi-polynomial, we can see that the minimum period is $6=\rho_{0}$.

\subsection{Via the Ehrhart theory}

We want to show via the Ehrhart theory that $\left|M\left(\mathcal{A}_{q}\right)\right|=\left|V \backslash \bigcup_{1<j<n} H_{j, q}\right|$ is a quasipolynomial in $q \in \mathbb{Z}_{>0}$. The Ehrhart theory is indeed useful for establishing that $\left|M\left(\mathcal{A}_{q}\right)\right|$ is a quasi-polynomial, and gives a geometric insight into its period. However, it does not seem to give information on the constituents of the quasi-polynomial.

For $j=1, \ldots, n$, let

$$
S_{j}:=\mathbb{Z} \cap\left\{x c_{j} \mid x \in[0,1)^{m}\right\}
$$

For example, for $c_{j}=(1, \ldots, 1)^{T} \in \mathbb{Z}^{m}$

$$
\min _{\substack{x \in[0,1)^{m} \\ x_{1}+\cdots+x_{m} \in \mathbb{Z}}}\left(x_{1}+\cdots+x_{m}\right)=0, \quad \max _{\substack{x \in[0,1)^{m} \\ x_{1}+\cdots+x_{m} \in \mathbb{Z}}}\left(x_{1}+\cdots+x_{m}\right)=m-1,
$$

and $S_{j}=\{0,1, \ldots, m-1\}$ for this $c_{j}$. Now define the additional "translated" hyperplanes

$$
H_{j}^{s_{j}}(q)=H_{c_{j}}^{s_{j}}(q):=\left\{x=\left(x_{1}, \ldots, x_{m}\right) \in \mathbb{R}^{m}: x c_{j}=s_{j} q\right\} \subset \mathbb{R}^{m}, \quad s_{j} \in S_{j},
$$

for $j=1, \ldots, n$, and consider the real hyperplane arrangement

$$
\mathcal{A}^{\text {deform }}(q)=\mathcal{A}_{C}^{\text {deform }}(q):=\left\{H_{j}^{s_{j}}(q): s_{j} \in S_{j}, 1 \leq j \leq n\right\} .
$$

For any positive integer $q$, we can express $\left|M\left(\mathcal{A}_{q}\right)\right|=\left|V \backslash \bigcup_{1 \leq j \leq n} H_{j, q}\right|$ as

$$
\begin{aligned}
\left|M\left(\mathcal{A}_{q}\right)\right| & =\left|\mathbb{Z}^{m} \cap[0, q)^{m} \backslash \cup \mathcal{A}^{\text {deform }}(q)\right| \\
& =\left|\mathbb{Z}^{m} \cap\left(q \times\left([0,1)^{m} \backslash \cup \mathcal{A}^{\text {deform }}\right)\right)\right|,
\end{aligned}
$$

where $\cup \mathcal{A}^{\operatorname{deform}_{(q)}}:=\bigcup_{H \in \mathcal{A}^{\text {deform }}(q)} H$ and $\mathcal{A}^{\text {deform }}:=\mathcal{A}^{\text {deform }}(1)$. 
Now, let us consider $[0,1)^{m} \backslash \cup \mathcal{A}^{\text {deform }}$ in (14). We see that $[0,1)^{m}$ is cut by the hyperplanes $H_{j}^{s_{j}}(1), s_{j} \in S_{j}, 1 \leq j \leq n$, into

$$
P^{O}\left(s_{1}, \ldots, s_{n}\right):=\left\{x \in[0,1)^{m}: s_{j}<x c_{j}<s_{j}+1,1 \leq j \leq n\right\},
$$

$\left(s_{1}, \ldots, s_{n}\right) \in S_{1}^{*} \times \cdots \times S_{n}^{*}=: S^{*}$, where $S_{j}^{*}:=S_{j} \cup\left\{\min S_{j}-1\right\}, 1 \leq j \leq n$. Therefore

$$
[0,1)^{m} \backslash \cup \mathcal{A}^{\text {deform }}=\bigsqcup_{\left(s_{1}, \ldots, s_{n}\right) \in S^{*}} P^{O}\left(s_{1}, \ldots, s_{n}\right)
$$

is a disjoint union. From (14) and (15), we obtain

$$
\left|M\left(\mathcal{A}_{q}\right)\right|=\sum_{\left(s_{1}, \ldots, s_{n}\right) \in S^{*}}\left|\mathbb{Z}^{m} \cap q P^{O}\left(s_{1}, \ldots, s_{n}\right)\right|=\sum_{\left(s_{1}, \ldots, s_{n}\right) \in S^{*}} i\left(P^{O}\left(s_{1}, \ldots, s_{n}\right), q\right),
$$

where $i\left(P^{O}\left(s_{1}, \ldots, s_{n}\right), q\right):=\left|\mathbb{Z}^{m} \cap q P^{O}\left(s_{1}, \ldots, s_{n}\right)\right|$.

It should be noted that $P^{O}\left(s_{1}, \ldots, s_{n}\right),\left(s_{1}, \ldots, s_{n}\right) \in S^{*}$, are not necessarily open in $\mathbb{R}^{m}$. However, by applying the Ehrhart theory to some faces of each nonempty $P^{O}\left(s_{1}, \ldots, s_{n}\right)$, we can show that $i\left(P^{O}\left(s_{1}, \ldots, s_{n}\right), q\right)$ is a quasi-polynomial of $q \in \mathbb{Z}_{>0}$ with degree $d=\operatorname{dim}\left(P^{O}\left(s_{1}, \ldots, s_{n}\right)\right)$ and the leading coefficient equal to the normalized volume of $P^{O}\left(s_{1}, \ldots, s_{n}\right)$. When $d=m$, the normalized volume is the same as the usual volume in $\mathbb{R}^{m}$. Therefore, we can conclude that the sum $\sum_{\left(s_{1}, \ldots, s_{n}\right) \in S^{*}} i\left(P^{O}\left(s_{1}, \ldots, s_{n}\right), q\right)=\left|M\left(\mathcal{A}_{q}\right)\right|$ is a quasi-polynomial of $q \in \mathbb{Z}_{>0}$ with degree $m$ and the leading coefficient $\sum \operatorname{vol}_{m}\left(P^{O}\left(s_{1}, \ldots, s_{n}\right)\right)=\operatorname{vol}_{m}\left([0,1)^{m}\right)=1$, where $\operatorname{vol}_{m}(\cdot)$ denotes the usual volume in $\mathbb{R}^{m}$.

Let us move on to the investigation into periods of the characteristic quasipolynomial $\left|M\left(\mathcal{A}_{q}\right)\right|, q \in \mathbb{Z}_{>0}$. From the above discussion, we see that a common multiple of periods of $i\left(P^{O}\left(s_{1}, \ldots, s_{n}\right), q\right),\left(s_{1}, \ldots, s_{n}\right) \in S^{*}$, is a period of $\left|M\left(\mathcal{A}_{q}\right)\right|$. Let $\bar{P}\left(s_{1}, \ldots, s_{n}\right)$ denote the closure of $P^{O}\left(s_{1}, \ldots, s_{n}\right)$. Define the denominator $\mathcal{D}\left(\mathcal{A}^{\text {deform }}\right)$ of $\mathcal{A}^{\text {deform }}$ by

$$
\begin{aligned}
\mathcal{D}\left(\mathcal{A}^{\mathrm{deform}}\right):= & \min \left\{q \in \mathbb{Z}_{>0}: \text { all } q \bar{P}\left(s_{1}, \ldots, s_{n}\right),\left(s_{1}, \ldots, s_{n}\right) \in S^{*},\right. \\
& \text { are integral polytopes }\} .
\end{aligned}
$$

The Ehrhart theory now implies that $\mathcal{D}\left(\mathcal{A}^{\text {deform }}\right)$ is a period of $\left|M\left(\mathcal{A}_{q}\right)\right|$.

Put $\tilde{C}:=\left(C, I_{m}\right)$, where $I_{m}$ is the $m \times m$ identity matrix. For $J \subseteq\{1, \ldots, n+m\}$, let $\tilde{C}_{J}$ denote the $m \times|J|$ submatrix of $\tilde{C}$ consisting of the columns corresponding to the elements of $J$. Then, in view of Cramer's formula, we see that $\mathcal{D}\left(\mathcal{A}^{\text {deform }}\right)$ divides

$$
\begin{aligned}
\rho_{\mathrm{E}}:= & \operatorname{lcm}\left\{\operatorname{det}\left(\tilde{C}_{J}\right): J \subset\{1, \ldots, n+m\}\right. \\
& \text { such that } \left.|J|=m \text { and } \operatorname{det}\left(\tilde{C}_{J}\right) \neq 0\right\} .
\end{aligned}
$$

Hence, the minimum period divides $\rho_{\mathrm{E}}$. For $J$ with $|J \cap\{n+1, \ldots, n+m\}|=h<$ $m$, the determinant $\operatorname{det}\left(\tilde{C}_{J}\right)$ equals an $(m-h) \times(m-h)$ minor of $C$ up to sign. Therefore, we can also write

$$
\rho_{\mathrm{E}}=\operatorname{lcm}\{\text { nonzero } j \text {-minors of } C, 1 \leq j \leq m\} .
$$


Now, recall the well-known fact that $\bar{e}(J):=e_{J, 1} \times \cdots \times e_{J, \ell(J)}$ is equal to the greatest common divisor of all the (nonzero) $\ell(J)$-minors of $C_{J}, J \neq \varnothing$, and note the relation $e(J)=e_{J, \ell(J)} \mid \bar{e}(J), J \neq \emptyset$. Then we can easily see from (11) and (16) that $\rho_{0} \mid \rho_{\mathrm{E}}$. Therefore, $\rho_{0}$ gives a tighter bound for the period of the characteristic quasipolynomial $\left|M\left(\mathcal{A}_{q}\right)\right|$ than $\rho_{\mathrm{E}}$.

In our working example (2), we have $\rho_{\mathrm{E}}=\operatorname{lcm}\{1,1,-2,-1,1,1,2,-1,3\}=6$ and thus $\rho_{0}=\rho_{\mathrm{E}}$. In general, if we obtain the characteristic quasi-polynomial by interpolation using $\rho_{\mathrm{E}}$ as a period and find that $\rho_{\mathrm{E}}$ happens to be the minimum period, then we know that $\rho_{0}=\rho_{\mathrm{E}}$.

\subsection{Characteristic polynomial of the real arrangement}

Let $\chi(\mathcal{A}, t)$ be the characteristic polynomial of the real hyperplane arrangement $\mathcal{A}=$ $\left\{H_{j}: 1 \leq j \leq n\right\}$, where $H_{j}=\left\{x \in \mathbb{R}^{m}: x c_{j}=0\right\}, 1 \leq j \leq n$.

Theorem 2.5 Let $\rho$ be a period of the quasi-polynomial $\left|M\left(\mathcal{A}_{q}\right)\right|$ and $q$ be a positive integer relatively prime to $\rho$. Then $\left|M\left(\mathcal{A}_{q}\right)\right|=\chi(\mathcal{A}, q)$.

Proof Choose $c \in \mathbb{Z}_{\geq 0}$ and $q^{\prime} \in \mathbb{Z}$ such that $q=\rho c+q^{\prime}$ and $1 \leq q^{\prime} \leq \rho$. By Theorem 2.4, there exist integers $\alpha_{0}, \ldots, \alpha_{m-1}$ such that $\left|M\left(\mathcal{A}_{k}\right)\right|=k^{m}+\alpha_{m-1} k^{m-1}+$ $\cdots+\alpha_{0}$ for all $k \in q^{\prime}+\rho \mathbb{Z}_{\geq 0}$. Since $q^{\prime}$ and $\rho$ are relatively prime, then by Dirichlet's theorem on arithmetic progressions (e.g., [14]), $q^{\prime}+\rho \mathbb{Z}_{\geq 0}$ contains an infinite number of primes. On the other hand, it is well known (e.g., [9], [16, (4.10)], [10, Theorem 3.2]) that, when $k$ is a sufficiently large prime, $\left|M\left(\mathcal{A}_{k}\right)\right|$ coincides with $\chi(\mathcal{A}, k)$. Recall that the characteristic polynomial $\chi(\mathcal{A}, t)$ is a monic polynomial of degree $\operatorname{dim}\left(\mathbb{R}^{m}\right)=m$. This implies that $\chi(\mathcal{A}, t)=t^{m}+\alpha_{m-1} t^{m-1}+\cdots+\alpha_{0}$ and thus $\chi(\mathcal{A}, q)=q^{m}+\alpha_{m-1} q^{m-1}+\cdots+\alpha_{0}=\left|M\left(\mathcal{A}_{q}\right)\right|$.

Remark 2.6 A result similar to (but less detailed than) Theorem 2.5 was obtained by Athanasiadis [2, Theorem 2.1]. His proof is essentially by the method of elementary divisors as in Section 2.1.

The argument in the proof of Theorem 2.5 implies that we can obtain the characteristic polynomial $\chi(\mathcal{A}, t)$ by counting $\left|M\left(\mathcal{A}_{q_{i}}\right)\right|=\left|\mathbb{Z}_{q_{i}}^{m} \backslash \bigcup_{1 \leq j \leq n} H_{j, q_{i}}\right|$ for an arbitrary set of $m$ distinct values $q_{1}, \ldots, q_{m}$ with $\operatorname{gcd}\left\{\rho, q_{i}\right\}=1(1 \leq i \leq m)$. Note that $q_{1}, \ldots, q_{m}$ need not be prime. When $q^{\prime}$ and $\rho$ are not relatively prime, $q^{\prime}+\rho \mathbb{Z}_{\geq 0}$ contains at most one prime (and this prime is not necessarily "sufficiently large"), so the above argument does not hold. For $q^{\prime}+\rho \mathbb{Z}_{\geq 0}$ with such $q^{\prime}$ 's, we obtain different polynomials than $\chi(\mathcal{A}, t)$. In our example (2), the constituent of the characteristic quasi-polynomial $(13)$ for $1+6 \mathbb{Z}_{\geq 0}$ and $5+6 \mathbb{Z}_{\geq 0}$, i.e., $\operatorname{gcd}\{6, q\}=1$, is the characteristic polynomial of $\mathcal{A}$. Thus $\chi(\mathcal{A}, t)=t^{2}-3 t+2=(t-1)(t-2)$.

\section{Periodicity of the intersection lattice}

In this section, we show that the intersection lattice $L_{q}=L\left(\mathcal{A}_{q}\right)$ (e.g., $[13,2.1]$, [15, Chap. 3, Exercise 56]) is periodic for large enough $q$. Let us begin with our working example to illustrate the periodicity of the intersection lattice $L_{q}$. 


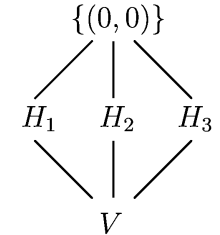

$\operatorname{gcd}\{6, q\}=1$

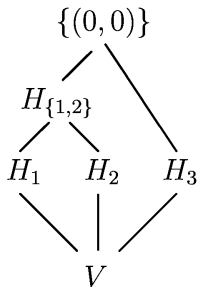

$\operatorname{gcd}\{6, q\}=2$

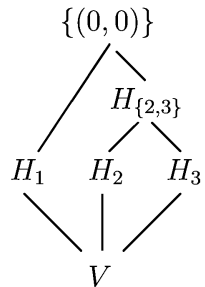

$\operatorname{gcd}\{6, q\}=3$

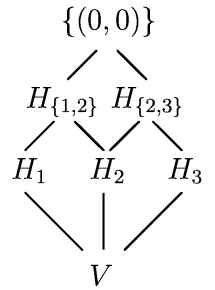

$\operatorname{gcd}\{6, q\}=6$

Fig. 1 Hasse diagrams of intersection lattices for $q \geq 4$

In our example (2), the "hyperplanes" $H_{1, q}, H_{2, q}, H_{3, q}$ were given in (3). For $q=1, V=\left\{\left([0]_{1},[0]_{1}\right)\right\}=H_{1,1}=H_{2,1}=H_{3,1}$. For $q=2, H_{1,2}=H_{2,2}$ and for $q=3, H_{2,3}=H_{3,3}$. These are the exceptions. From $q=4$ on, we have the periodicity of the intersection lattice - the lattice of $H_{J, q}=\bigcap_{j \in J} H_{j, q}, J \subseteq\{1,2,3\}$, ordered by reverse inclusion. First, it is easily seen that for $q \geq 4, H_{j, q}, j=1,2,3$, are distinct, proper subsets of $V$. Furthermore, for $q \geq 4$,

$$
\begin{aligned}
& H_{\{1,2\}, q}= \begin{cases}\left\{\left([0]_{q},[0]_{q}\right)\right\}, & q: \text { odd }, \\
\left\{\left([0]_{q},[0]_{q}\right),\left(\left[\frac{q}{2}\right]_{q},\left[\frac{q}{2}\right]_{q}\right)\right\}, & q: \text { even, }\end{cases} \\
& H_{\{2,3\}, q}= \begin{cases}\left\{\left([0]_{q},[0]_{q}\right)\right\}, & 3 \times q, \\
\left\{\left([0]_{q},[0]_{q}\right),\left(\left[\frac{q}{3}\right]_{q},\left[\frac{2 q}{3}\right]_{q}\right),\left(\left[\frac{2 q}{3}\right]_{q},\left[\frac{q}{3}\right]_{q}\right)\right\}, & 3 \mid q,\end{cases}
\end{aligned}
$$

and $H_{\{1,3\}, q}=H_{\{1,2,3\}, q}=\left\{\left([0]_{q},[0]_{q}\right)\right\}$. We see that the intersection lattice for this example is periodic and has the period 6 for $q \geq 4$. The Hasse diagrams for the four types of the intersection lattices are illustrated in Fig. 1. In Fig. 1, the subscript $q$ is omitted for simplicity.

Let $J=\left\{j_{1}, \ldots, j_{k}\right\}, 1 \leq j_{1}<\cdots<j_{k} \leq n, 1 \leq k \leq n$, be a nonempty subset of $\{1, \ldots, n\}$. We write the Smith normal form of $C_{J} \in \operatorname{Mat}_{m \times k}(\mathbb{Z})$ as

$$
\begin{aligned}
& S_{J} C_{J} T_{J}=\operatorname{diag}\left(\left\{e_{J, 1}, \ldots, e_{J, \ell(J)}\right\} ; m, k\right)=: \tilde{E}_{J}, \\
& \ell(J)=\operatorname{rank} C_{J}, \quad e_{J, 1}, \ldots, e_{J, \ell(J)} \in \mathbb{Z}_{>0}, \quad e_{J, 1}\left|e_{J, 2}\right| \cdots \mid e_{J, \ell(J)} .
\end{aligned}
$$

As in Section 2.1, we write $e(J)=e_{J, \ell(J)}$, the largest elementary divisor of $C_{J}$.

Let $\rho_{0}$ be the least common multiple of all $e(J)$ 's with $1 \leq|J| \leq \min \{m, n\}$ as in (11). Furthermore, define

$$
q_{0}:=\max _{\emptyset \neq J \subseteq\{1, \ldots, n\}} \min _{S_{J}} \max \left\{|u|: u \text { is an entry of } S_{J} C \text { or } C\right\},
$$

where the minimization is over all possible choices of $S_{J}$ in (17) for each fixed $J$.

We are now in a position to state the main theorem of this section.

Theorem 3.1 Let $J$ be an arbitrary nonempty subset of $\{1, \ldots, n\}$. Suppose $q, q^{\prime} \in$ $\mathbb{Z}_{>0}$ satisfy $q, q^{\prime}>q_{0}$ and $\operatorname{gcd}\left\{\rho_{0}, q\right\}=\operatorname{gcd}\left\{\rho_{0}, q^{\prime}\right\}$. Then, for any $j \in\{1, \ldots, n\}$, we have that $H_{j, q} \supseteq H_{J, q}$ if and only if $H_{j, q^{\prime}} \supseteq H_{J, q^{\prime}}$. 
When $j \in J$, the theorem is trivially true.

In proving Theorem 3.1, we need the following proposition. Regard $V=\mathbb{Z}_{q}^{m}$ as a $\mathbb{Z}_{q}$-module. Let $V^{*}$ be the $\mathbb{Z}_{q}$-module consisting of the linear forms on $V$. For any $A \subseteq V$, we denote by $I(A)$ the set of linear forms vanishing on $A$ :

$$
I(A)=\left\{\alpha \in V^{*}: \alpha(x)=0 \text { for all } x \in A\right\} .
$$

Also, for any $B \subseteq V^{*}$, let $V(B)$ stand for the set of points at which each linear form in $B$ vanishes:

$$
V(B)=\{x \in V: \alpha(x)=0 \text { for all } \alpha \in B\} .
$$

Evidently, $I(A)$ and $V(B)$ are submodules of $V^{*}$ and $V$, respectively.

Proposition 3.2 For any $B \subseteq V^{*}$, we have $I(V(B))=\langle B\rangle$, where $\langle B\rangle$ denotes the submodule of $V^{*}$ spanned by $B$.

Proof It suffices to show that $I(V(B))=B$ for any submodule $B$ of $V^{*}$. It is trivially true that $I(V(B)) \supseteq B$, so we will prove $I(V(B)) \subseteq B$.

Let $C_{q}(B) \in \operatorname{Mat}_{m \times k}\left(\mathbb{Z}_{q}\right), k=|B|$, be the coefficient matrix of $B$. We can find an integral matrix $C(B) \in \operatorname{Mat}_{m \times k}(\mathbb{Z})$ whose $q$-reduction is $C_{q}(B)$, i.e., $[C(B)]_{q}=$ $C_{q}(B)$. Now, let $e_{1}\left|e_{2}\right| \cdots \mid e_{\ell}, \ell=\operatorname{rank} C(B)$, be the elementary divisors of $C(B)$. In $\mathbb{Z}_{q}$, we then have $C_{q}(B)$ is equivalent to

$$
\operatorname{diag}\left(\left\{\left[e_{1}\right], \ldots,\left[e_{\ell^{\prime}}\right]\right\} ; m, k\right) \in \operatorname{Mat}_{m \times k}\left(\mathbb{Z}_{q}\right),
$$

where $\left[e_{1}\right], \ldots,\left[e_{\ell^{\prime}}\right] \in \mathbb{Z}_{q} \backslash\{0\}, \ell^{\prime} \leq \ell$. Here, we are writing $[\cdot]$ for $[\cdot]_{q}$ for simplicity. We can choose $C(B)$ in such a way that $\ell^{\prime}=\ell$, and we decide to do so. From (18) we see that we can assume $B$ is spanned by $\left[e_{1}\right] y_{1}, \ldots,\left[e_{\ell}\right] y_{\ell}$ after a suitable coordinate change, where $\left\{y_{1}, \ldots, y_{\ell}, y_{\ell+1}, \ldots, y_{m}\right\}$ is a basis of $V^{*}$. It follows that $V(B)$ is spanned by

$$
\begin{gathered}
p_{1}=\left(\left[q / d_{1}(q)\right], 0, \ldots, 0\right), \ldots, p_{\ell}:=(\underbrace{0, \ldots, 0}_{\ell-1},\left[q / d_{\ell}(q)\right], 0, \ldots, 0), \\
p_{\ell+1}:=(\underbrace{0, \ldots, 0}_{\ell}, 1,0, \ldots, 0), \ldots, p_{m}:=(0, \ldots, 0,1)
\end{gathered}
$$

with $d_{j}(q)=\operatorname{gcd}\left\{e_{j}, q\right\}, 1 \leq j \leq \ell$.

Now, take an arbitrary $\alpha=\left[a_{1}\right] y_{1}+\cdots+\left[a_{m}\right] y_{m} \in I(V(B))=I\left(p_{1}, \ldots, p_{m}\right)$ with $\left[a_{1}\right], \ldots,\left[a_{m}\right] \in \mathbb{Z}_{q}$. Then we have $0=\alpha\left(p_{1}\right)=\left[q a_{1} / d_{1}(q)\right]$, so $a_{1}=$ $r_{1} d_{1}(q)$ for some $r_{1} \in \mathbb{Z}$. This implies $\left[a_{1}\right]=\left[r_{1}\right]\left[d_{1}(q)\right]=\left[r_{1}^{\prime}\right]\left[e_{1}\right]$ with $\left[r_{1}^{\prime}\right]:=$ $\left[r_{1}\right]\left[e_{1} / d_{1}(q)\right]^{-1} \in \mathbb{Z}_{q}$, where $\left[e_{1} / d_{1}(q)\right]^{-1}$ exists because $\operatorname{gcd}\left\{e_{1} / d_{1}(q), q\right\}=1$. Similarly, for each $j=2, \ldots, \ell$, we have $\left[a_{j}\right]=\left[r_{j}^{\prime}\right]\left[e_{j}\right]$ for some $\left[r_{j}^{\prime}\right] \in \mathbb{Z}_{q}$. Moreover, for $j=\ell+1, \ldots, m$, we obtain $0=\alpha\left(p_{j}\right)=\left[a_{j}\right]$. Therefore, we have $\alpha=\left[r_{1}^{\prime}\right]\left[e_{1}\right] y_{1}+\cdots+\left[r_{\ell}^{\prime}\right]\left[e_{\ell}\right] y_{\ell} \in B$, and the proof is complete.

Proof of Theorem 3.1 Without loss of generality, we may assume $j=1$. Let $\left[S_{J}\right]_{q},\left[C_{J}\right]_{q},\left[T_{J}\right]_{q}$ and $\left[\tilde{E}_{J}\right]_{q}$ be the $q$-reductions of $S_{J}, C_{J}, T_{J}$ and $\tilde{E}_{J}$ in (17), respectively. 
First, we know by Proposition 3.2 that $H_{1, q} \supseteq H_{J, q}$ if and only if $\left[c_{1}\right]_{q}$ lies in the column space of $\left[C_{J}\right]_{q}$ in $\mathbb{Z}_{q}^{m}$. Since $S_{J}^{-1}$ and $T_{J}^{-1}$ exist in $\operatorname{Mat}_{m \times m}(\mathbb{Z})$ and $\operatorname{Mat}_{k \times k}(\mathbb{Z})$, respectively, the latter condition is equivalent to $\left[c_{1}\right]_{q}$ being in the column space of $\left[C_{J}\right]_{q}\left[T_{J}\right]_{q}=\left[S_{J}^{-1}\right]_{q}\left[\tilde{E}_{J}\right]_{q}$, which in turn is equivalent to $\left[S_{J}\right]_{q}\left[c_{1}\right]_{q}$ being in the column space of $\left[\tilde{E}_{J}\right]_{q}$ in $\mathbb{Z}_{q}^{m}$.

Next, let us paraphrase the above condition in $\mathbb{Z}_{q}^{m}$ as a condition in $\mathbb{Z}^{m}$. The condition holds if and only if $S_{J} c_{1} \in \mathbb{Z}^{m}$ is in the column space of $\left(\tilde{E}_{J}, q I_{m}\right) \in$ $\operatorname{Mat}_{m \times(k+m)}(\mathbb{Z})$ in $\mathbb{Z}^{m}$. Noting that $e_{J, j} \mathbb{Z}+q \mathbb{Z}=d_{J, j}(q) \mathbb{Z}$ with $d_{J, j}(q)=$ $\operatorname{gcd}\left\{e_{J, j}, q\right\}, 1 \leq j \leq \ell(J)$, we see that the condition holds if and only if $S_{J} c_{1}$ is in the column space of $\operatorname{diag}\left(d_{J, 1}(q), \ldots, d_{J, \ell(J)}(q), q, \ldots, q\right) \in \operatorname{Mat}_{m \times m}(\mathbb{Z})$. Since the absolute value of each entry of $S_{J} c_{1} \in \mathbb{Z}^{m}$ is less than $q$, the condition is equivalent to $S_{J} c_{1}$ being in the column space of

$$
\operatorname{diag}\left(\left\{d_{J, 1}(q), \ldots, d_{J, \ell(J)}(q)\right\} ; m, \ell(J)\right) \in \operatorname{Mat}_{m \times \ell(J)}(\mathbb{Z}) .
$$

Now, since the absolute value of each entry of $S_{J} c_{1}$ is less than $q^{\prime}$ as well, the preceding argument holds true also for $q^{\prime}$. Moreover, we see from (12) that $d_{J, j}(q)=$ $d_{J, j}\left(q^{\prime}\right)$ for $j=1, \ldots, \ell(J)$. Thus (19) remains the same when $q$ is replaced by $q^{\prime}$. Therefore, we obtain the desired result.

Our assumption (1) implies that $H_{j, q} \nsupseteq H_{\emptyset, q}=V, 1 \leq j \leq n$, for all $q>q_{0}$. From this observation and Theorem 3.1, it follows immediately that $L_{q}=L\left(\mathcal{A}_{q}\right)$ for $q>q_{0}$ is periodic in $q$ with a period $\rho_{0}$.

Corollary 3.3 The intersection lattice $L_{q}=L\left(\mathcal{A}_{q}\right)$ is periodic in $q>q_{0}$ with a period $\rho_{0}$ :

$$
L_{q+s \rho_{0}} \simeq L_{q} \text { for all } q>q_{0} \text { and } s \in \mathbb{Z}_{\geq 0}
$$

Finally, we make a remark on the coarseness of the intersection lattices for different $q$ 's. In Fig. 1 we see that the intersection lattice for the case $\operatorname{gcd}\{6, q\}=6$ is the most detailed and that the coarseness is nested according to the divisibility of $\operatorname{gcd}\{6, q\}$. This observation can be generally stated as follows.

Proposition 3.4 Let $I, J \subseteq\{1, \ldots, n\}$ and suppose that $H_{I, q}=H_{J, q}$ for some $q>q_{0}$. Then $H_{I, q^{\prime}}=H_{J, q^{\prime}}$ for every $q^{\prime}>q_{0}$ such that $\operatorname{gcd}\left\{\rho_{0}, q^{\prime}\right\} \mid \operatorname{gcd}\left\{\rho_{0}, q\right\}$.

Proof It suffices to show that for any $i \in I$, if $\left[c_{i}\right]_{q}$ lies in the column space of $\left[C_{J}\right]_{q}$ in $\mathbb{Z}_{q}^{m}$, then $\left[c_{i}\right]_{q^{\prime}}$ lies in the column space of $\left[C_{J}\right]_{q^{\prime}}$ in $\mathbb{Z}_{q^{\prime}}^{m}$. Without loss of generality, take $i=1$ and assume that $\left[c_{1}\right]_{q}$ lies in the column space of $\left[C_{J}\right]_{q}$ in $\mathbb{Z}_{q}^{m}$. Then $S_{J} c_{1}$ is in the column space of (19). Now, because $\operatorname{gcd}\left\{\rho_{0}, q^{\prime}\right\} \mid \operatorname{gcd}\left\{\rho_{0}, q\right\}$ by assumption, we can see from (12) that $d_{J, j}\left(q^{\prime}\right) \mid d_{J, j}(q), 1 \leq j \leq \ell(J)$. This implies that $S_{J} c_{1}$ is in the column space of (19) with $q$ replaced by $q^{\prime}$. Therefore, $\left[c_{1}\right]_{q^{\prime}}$ lies in the column space of $\left[C_{J}\right]_{q^{\prime}}$ in $\mathbb{Z}_{q^{\prime}}^{m}$. 


\section{References}

1. Athanasiadis, C.A.: Characteristic polynomials of subspace arrangements and finite fields. Adv. Math. 122, 193-233 (1996)

2. Athanasiadis, C.A.: Extended Linial hyperplane arrangements for root systems and a conjecture of Postnikov and Stanley. J. Algebr. Comb. 10, 207-225 (1999)

3. Athanasiadis, C.A.: Generalized Catalan numbers, Weyl groups and arrangements of hyperplanes. Bull. Lond. Math. Soc. 36, 294-302 (2004)

4. Athanasiadis, C.A.: A combinatorial reciprocity theorem for hyperplane arrangements. arXiv:math.CO/0610482v1; Can. Math. Bull. (to appear)

5. Beck, M., Robins, S.: Computing the Continuous Discretely: Integer-Point Enumeration in Polyhedra. Springer, Berlin (2007)

6. Beck, M., Zaslavsky, T.: Inside-out polytopes. Adv. Math. 205, 134-162 (2006)

7. Björner, A., Ekedahl, T.: Subspace arrangements over finite fields: cohomological and enumerative aspects. Adv. Math. 129, 159-187 (1997)

8. Blass, A., Sagan, B.: Characteristic and Ehrhart polynomials. J. Algebr. Comb. 7, 115-126 (1998)

9. Crapo, H., Rota, G.-C.: On the Foundations of Combinatorial Theory: Combinatorial Geometries. MIT Press, Cambridge (1970) (preliminary edn.)

10. Kamiya, H., Orlik, P., Takemura, A., Terao, H.: Arrangements and ranking patterns. Ann. Comb. 10, 219-235 (2006)

11. Kamiya, H., Takemura, A., Terao, H.: The characteristic quasi-polynomials of the arrangements of root systems and mid-hyperplane arrangements. In preparation

12. McAllister, T.B., Woods, K.M.: The minimum period of the Ehrhart quasi-polynomial of a rational polytope. J. Comb. Theory Ser. A 109, 345-352 (2005)

13. Orlik, P., Terao, H.: Arrangements of Hyperplanes. Springer, Berlin (1992)

14. Serre, J.-P.: Cours d'Arithmétique. Presses Universitaires de France, Paris (1970)

15. Stanley, R.: Enumerative Combinatorics, vol. I. Cambridge University Press, Cambridge (1997)

16. Terao, H.: The Jacobians and the discriminants of finite reflection groups. Tohoku Math. J. 41, 237247 (1989) 